\title{
Impact of chemical elements on heart failure progression in coronary heart disease patients
}

\author{
Karaskov Alexander, Kamenskaya Oksana*, Levicheva Elena, Loginova Irina, Okuneva Galina, \\ Cherniavsky Aleksander, Kliver Evgeni, Volkov Alexander
}

Federal State Institution Academician E.N.Meshalkin Novosibirsk State Research Institute of Circulation Pathology Rusmedtechnology, Novosibirsk, Russian Federation; "Corresponding Author: physiolog@,ngs.ru

Received 31 January 2011; revised 20 April 2011; accepted 27 April 2011.

\section{ABSTRACT}

Background. The high prevalence, poor prognosis of patients with coronary heart disease with chronic heart failure determine the relevance of the study pathophysiological and molecular mechanisms of this pathology. Researches of the trace element metabolism in the myocardium are scarce. With this in mind, an attempt was made to analyze the relationship of macro and trace elements metabolism with the functional state of the myocardium in coronary heart disease patients against the background of chronic heart failure progression. Methods and Results. To study the content of the chemical elements ( $\mathrm{S}, \mathrm{K}, \mathrm{Ca}, \mathrm{Cr}, \mathrm{Fe}, \mathrm{Ni}, \mathrm{Cu}, \mathrm{Zn}$, $\mathrm{Se}, \mathrm{Rb}, \mathrm{Sr}$ ) in the myocardium of 43 patients with coronary heart disease, use was made of $X$-ray fluorescence with synchrotron radiation. While doing autopsy, 43 samples of left ventricle myocardium were taken off the cardiac callosity. Myocardium samples were subjected to histological examination. Dynamics of macro and trace elements content in the myocardium reflects the development of energy deficiency and disorders of myocardial microcirculation with a decrease of systolic myocardial function. Structural/functional disorders in the myocardium of the left ventricle of patients with coronary heart disease that accompany the progression of chronic heart failure are associated with profound changes of metabolic processes in heart muscle. Conclusions. The structural/ functional changes accompanying chronic heart failure progression are associated with wide variations of metabolic processes in the myocardium, which could be evaluated by the content of chemical elements in tissue.
Keywords: Heart Failure; Tissue; Myocardium

\section{INTRODUCTION}

Chronic heart failure (CHF) is a pathophysiological syndrome caused by structural or functional cardiac disorders that affect the ventricular function to pump and eject the blood [1]. In spite of recent advances in medicine, the prevalence of hospital admissions and lethal outcomes in CHF patients remains high. Every year cardiovascular diseases take 4.3 million human lives in Europe and over 2 million cardiac patients annually die in EU countries [2].

A coronary heart disease (CHD) is the primary cause of CHF. At present a progression of cardiac insufficiency is considered as the consequence of an imbalance in the biochemical mechanisms system [3]. Direct damaging actions of ischemia, hypertension or inflammation are considered to be the main causes of such changes in CHD patients.

Adequate metabolism is the main criterion of viability of every cell in the organism. Therefore, any changes in the concentration of chemical elements might serve as a diagnostic marker of pathological changes in tissue.

A biochemical imbalance, which frequently accompanies CHD, leads to progression of the left ventricular dysfunction by speeding up myocardium remodeling processes [4].

The contractility of the myocardium is maintained by the appropriate electrolytic composition of cellular and intercellular substances, as well as by enzymatic activity of biochemical processes [5]. Metals are active enzyme centers; therefore, determining the content of metals in the myocardial tissue might shed some light on adequate or inadequate metabolism of the heart.

However, the studies relating to the content of macroand microelements in the tissue of various organs (including the myocardium) are largely conducted on animals. Humans are mainly involved when determining 
the content of macro and trace elements in blood serum in patients with different pathologies [6,7].

These studies are frequently incommensurable because the authors use different methods for measuring the content of chemical elements and due to the fact that blood being a common collector receives all products of organism metabolism. Therefore, relating a particular imbalance in the content of macro or trace elements in blood to the pathology of a specific organ is a challenge. Unfortunately, there are very few studies on metabolism of human organs (excluding biochemical studies) but this problem attracts considerable attention of researchers. Of special interest is the investigation of metabolism of the heart affected by a coronary disease, because the morbidity of this pathology is rather high. With this in mind, an attempt was made to analyze the relationship of mineral metabolism with the functional state of the myocardium in CHD patients against the background of chronic heart failure progression.

\section{METHODS}

\subsection{Patients}

We studied 43 patients with CHD (66\% males, 34\% females, mean age $61 \pm 1.6$ years). Coronary heart disease was diagnosed in the patients on the basis of medical history, physical findings, electrocardiography, echocardiography and coronary angiography. In accordance with New York Heart Association classification 24\% of patients fell under functional class (FC) II, 62\% - FC III and 14\% - FC IV. All CHD patients' case history included myocardial infarction of the LV with different periods of duration, and all of them died at the hospital as a result of heart failure progression.

\subsection{Tissue Samples}

Samples from heart muscle were obtained from 43 autopsies taken from CHD patients. 15 samples of LV myocardium belonging to people who died as a result of road accidents and who had no vascular pathologies were used as a control group. The post-mortem time from death to autopsy ranged from 12 to $24 \mathrm{~h}$. Left ventricle (LV) myocardium was taken off the cardiac callosity.

Myocardium samples for histological examination were excised from the anterior, posterior and apical parts of $\mathrm{LV}$, preserved in $10 \%$ formaline and embedded in paraffin. $5 \mu \mathrm{m}$-thick slices were obtained by using Microm HM550 microtome and stained with haematoksylin-eosin making use of van Gieson's method, with the elastic finished by combined application of orceine. PAS-reaction and Gomori one-step trichrome stain were also applied. Plain histology and morphometric studies were done by means of a microscope-based set which included a ZEISS optical microscope, an AxioCam MRc digital videocamera and a Pentium-4 computer. Measurements were performed by using an AxioVisio system at 400x magnification, with the microscope calibrated on a regular basis. The following morphological criteria were chosen to be used in the study: muscle fiber diameter, muscle tissue area, number of nuclei with the calculation of their average and total area, as well as nuclear-cytoplasmic index.

\subsection{Chemical Element Analysis}

The measurements were carried out at the experimental station of X-ray fluorescence elemental analysis (Budker Institute of Nuclear Physics SB RAS, storage ring VEPP-3). The masses of samples varied from 3 to 8 mg (wet tissue). The sample preparation was following: specimens were drying on fluoroplastic films overnight under the room temperature, samples were kept under the weight to get more flat surface of the sample. The coefficient of the loss of weight was from 1.7 to 2.0. Thus, the mass of sample analyzed was from 1.5 to 4.7 mg (dry tissue). Than, the samples were placed between two Mylar X-ray films (thickness of $2.5 \mu \mathrm{m}$ ) and clamped by the Teflon rings. Elemental concentrations in the samples of myocardium were calculated by the external standard method. Two certified reference materials (CRM) were used: NIES \#6 Mussel and NIST 1577 Bovine Liver. Chemical elements (CE) detected: S, K, Ca, $\mathrm{Cr}, \mathrm{Fe}, \mathrm{Ni}, \mathrm{Cu}, \mathrm{Zn}, \mathrm{Se}, \mathrm{Rb}, \mathrm{Sr}$. The content of $\mathrm{CE}$ was measured at $\mu \mathrm{g}$ per $1 \mathrm{~g}$ of tissue.

\subsection{Echocardiography Evaluation}

Based on the life-time ECG data obtained 2 - 5 days before the patient's death, some structural/functional parameters of the heart were measured and analyzed. The end-diastolic dimension of the left ventricle (EDD $\mathrm{LV}, \mathrm{cm}$ ), end-systolic and end-diastolic volumes of the left ventricle (ESV and EDV LV, ml) and ejection fraction of the left ventricle (EF LV, \%) were determined. Also calculated were myocardium mass (MM LV, g) and relative thickness of the left ventricle wall (RTW LV, rel. units). The ECG cardiometric data of healthy males of the same age were used for comparison.

\subsection{Statistical Analysis}

Statistical analysis was performed using the STATISTICA 6.1. The differences between the levels of the chemical elements in the myocardium of CHD patients with normal and low LV ejection fraction were tested by Mann-Whitney U test. Correlations between the levels of 
the chemical elements were calculated using Spearman rank correlation analysis. Value $\mathrm{p}<0.05$ was considered statistically significant.

\section{RESULTS AND DISCUSSION}

With CHF progressing, the myocardium undergoes structural/functional changes known as myocardial remodeling. This process includes myocardium hypertrophy, its dysfunction and death of cardiomyocytes, as well as alteration of the cardiac extracellular matrix, often referred to as myocardial fibrosis [8]. A systolic dysfunction evaluated primarily by LV EF is at present regarded as an independent predictor of CHF patients' diagnosis [9].

Undoubtedly, CHF progression is accompanied by both the systolic and diastolic dysfunction, with the diastole being an earlier and more vulnerable target and preceding the impairment of the systolic function. However, choosing the systolic rather than diastolic function of $\mathrm{LV}$ as a predictor of CHF prognosis is associated with the problem of early diagnostics of the diastolic dysfunction, since it is practically insidious [10,11].

Our ECG data show that as CHF progresses, by the example of a decreased EF, increased end volumes of LV, both systolic and diastolic ones, as well as a thinned myocardium of LV are observed (Figure 1).

An ischemic damage of myocardium followed by the development of a large-sized area of cicatrical tissue in the LV wall turning into aneurismal thinning of LV stimulates compensatory hypertrophy of myocardium both in the percicatrical area and in the entire muscle on a whole. With the coronary flow compromised, which is typical of CHD, hypertrophic cardiomyocytes start losing their hyperplastic intracellular structures (myofibrils, mitochondria) and get mummified, while some part of them is undergoing necrodystrophic and apoptotic changes. The morphological pattern of cardiac insufficiency is characterized by the presence of areas of dead cardiomyocytes, structural abnormalities of viable cardiomyocytes and fibrosis progression $[8,12]$.

Our evaluation of morphometric properties indicated that an average diameter of myocardial muscle fibers of $\mathrm{LV}$ in CHD patients $(9.8 \pm 2.2 \mu \mathrm{m})$ was nearly the same as that of healthy ones $(9.2 \pm 1.8, \mathrm{p}>0.05)$. At the same time the histogram of muscle fiber number distribution depending on their diameter demonstrated a decrease in the average-diameter fibers in CHD patients and an increase in extreme values (atrophic and hypertrophic fibers) as compared to the norm (Figure 2). Such a wide variability of fibers in thickness in CHD patients indicates that the compensatory hypertrophy stage starts wearing off and changing to a decompensation stage with muscle fiber atrophy.
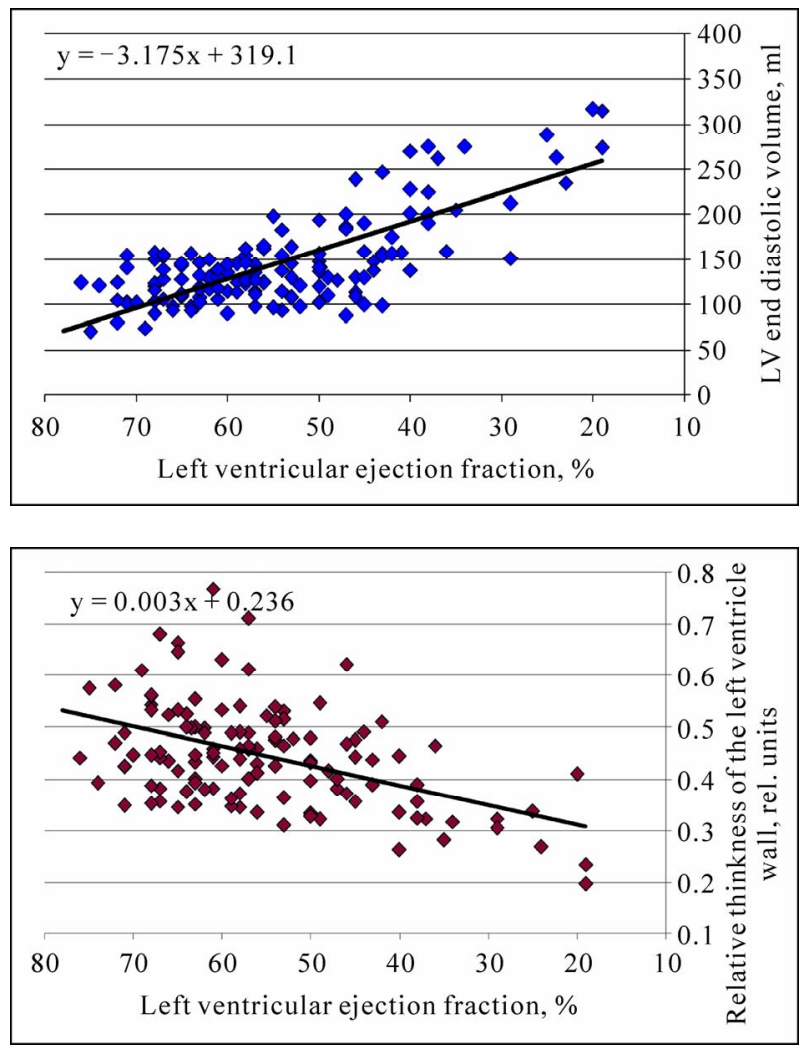

Figure 1. Structural/functional parameters of LV as cardiac insufficiency progresses in CHD patients.

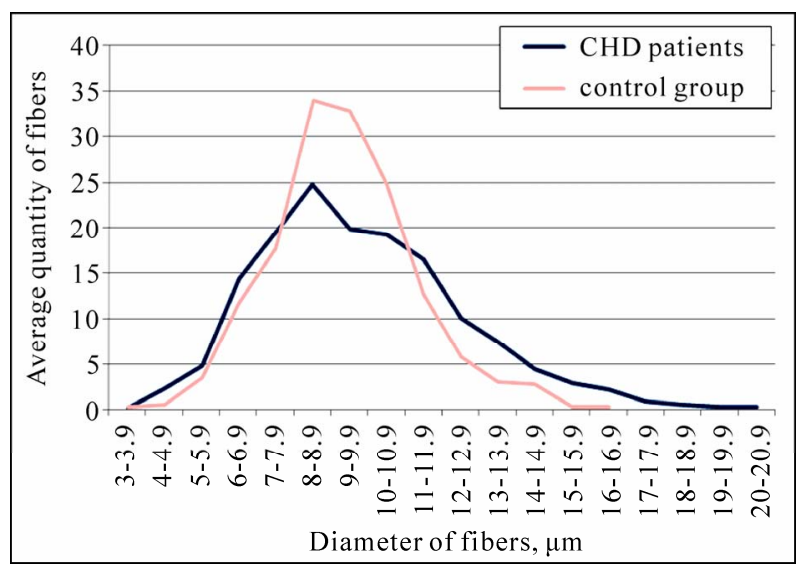

Figure 2. Relationship between quantity of muscle fibers and their diameter in LV in CHD patients and control group.

The analysis of the relationship between the number of cardiomyocyte nuclei and their area in LV myocardium revealed that in CHD patients the histogram of nuclei area distribution was shifted towards smaller structures. This evidences an earlier process of nuclear material depletion as compared with other intracellular structures, i.e. an atrophic and apoptotic focus of myocardial parenchymal structures depression in addition to their dysmetabolic necrosis in the case of ischemia. 
Histological symptoms of myocardial ischemia also include microcirculatory disorders manifesting themselves as non-uniform blood filling of myocardium capillaries, microhemorrhages. Nickel presents a vasoactive endogenic agent which might play an important role in this pathogenesis. This CE is assigned mainly a toxic effect on tissues [13]. However, our data are at variance with the categoricity of this point of view. In our study, as cardiac insufficiency progressed, the content of $\mathrm{Ni}$ decreased from $0.93 \mu \mathrm{g} / \mathrm{g}$ of tissue, with average value of $\mathrm{EF}$ equal to $63.5 \%$, to $0.35 \mu \mathrm{g} / \mathrm{g}$ of tissue, with average EF equal to $31.2 \%$. In our earlier study we demonstrated that Ni deficit reduced the possibility of angiogenesis in the conditions of compensatory hypertrophy development, which manifested itself as a low number of intramyocardial arteries [14]. A statistically significant direct correlation relationship has been found to exist between a relative thickness of the $\mathrm{LV}$ wall and $\mathrm{Ni}$ content $(r=0.76 ; p<0.01)$, which also demonstrates a considerable contribution of $\mathrm{Ni}$ to the development of the microcirculatory bed of LV myocardium. Nickel deficit has little effect on protein metabolism, however, it induces noticeable shifts in carbohydrate and lipid metabolism, thus suggesting that $\mathrm{Ni}$ influences energy exchange in the myocardium [15].

Chrome has a great impact on energy processes, since its main biological role in a human organism is connected with the regulation of carbohydrate and lipid metabolism, as well as with maintenance of tolerance to glucose $[16,17]$. Chrome is also important for normal metabolism of fats and its shortage would undoubtedly result in excess weight, obesity. Chrome is regarded as an activator of a number of enzymes [18]. Chrome concentration decreases when a patient is under stress (protein starvation, physical load, hypoxia, etc.) because of its increased discharge with urine. Perhaps, hypoxic stress, when cardiac insufficiency progresses, is a trigger for $\mathrm{Cr}$ deficit build-up in the myocardium, which further contributes to disruption of energy processes in CHD patients' myocardium (Figure 3).

Cardiac insufficiency progression is accompanied by the development of myocardial fibrosis, which originates not only in the areas of old myocardial infarction but in the non-involved zones as well. As LV contractility reduces, an increase in $\mathrm{S}$ concentration is observed in its myocardium, which might be associated with the increase in the content of connective tissue, because the latter is known to consist of mainly sulphide-bearing amino acids (Figure 4).

The same dynamics is typical for Fe content, the concentration of which increases, as the ejection fraction reduces. A statistically significant correlation of a moderately pronounced nature $(r=0.43: p<0.01)$ can be

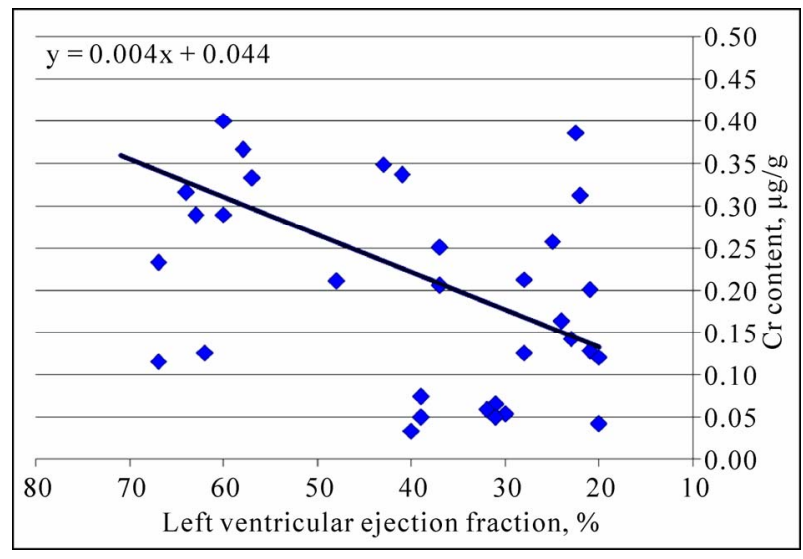

Figure 3. Dynamics of $\mathrm{Cr}$ content as cardiac insufficiency progresses in CHD patients.

observed between the contents of $\mathrm{S}$ and $\mathrm{Fe}$, which bears witness to uniformity of processes related to the imbalance of these elements. Diffuse propagation of connective tissue strengthens myocardium stiffness, which is a contributory factor not only for systolic but also diastolic dysfunction of LV [19].

Thus, an increase in $\mathrm{S}$ and $\mathrm{Fe}$ content demonstrates an aggravation of rigid processes in LV myocardium, as cardiac insufficiency progresses. Indeed, the morphological picture of a "rigid" myocardium with a low EF as compared to the myocardium with a normal EF has its own features. According to our data in chronic CHD patients there appear signs of dystrophia, necrobiosis and necrosis in different parts of the myocardium. In the area of necrotic loci there develop small scars, stromal collapse, coarsening of argyrophilic skeleton, with a scar elaborated without apparent cellular proliferation. In the cases of death as a result of incremental chronic cardiovascular insufficiency, myotomes in the state of contactures are few and far between, while partial disintegration of myofibrils predominates. Alongside with fresh necroses of myocytes, numerous cellular infiltrates consisting of macrophages and fibroblasts can be observed in the myocardium. This "patchy" picture of myocardium composed of muscle fibers with a different degree of dystrophy and necrobiosis, with dead myotomes replaced by connective tissue, testifies that the myocardium is subject to continuous and repeated injuries, with the connective tissue volume increasing in a gradual manner.

As the connective tissue increases, the muscle fibers gradually elongate, myocardium contractility reduces, LV cavity dilates and its walls get thinned (see Figure 1).

Insufficiency of $\mathrm{Cu}$ contributes to $\mathrm{Fe}$ accumulation in the tissue because it is impossible to borrow it from the tissue depot [20]. Indeed, as cardiac insufficiency progresses, $\mathrm{Cu}$ content in LV myocardium drops considerably 
(Figure 5). Copper deficit is a defective synthesis of collagen. Faulty synthesis of collagen and elastin in particular manifests itself as profuse internal hemorrhage caused by the formation of aneurisms in large arteries. A failure of those compounds that are responsible for tissue bridging is considered to be the main biochemical defect typical for this pathology, therefore $\mathrm{Cu}$ deficit leads to degeneration and fibrosis of the myocardium.

Many researchers suggest that cardiac insufficiency development is associated with interrelations between $\mathrm{Cu}$ and $\mathrm{Zn}$ [21-23]. A decrease in $\mathrm{Cu}$ concentration is more expressed as compared with that of $\mathrm{Zn}$, and as our data demonstrate, with LV myocardium contractility lowered, the $\mathrm{Zn} / \mathrm{Cu}$ proportion is increased nearly by 1.5 times at the expense of a decrease in $\mathrm{Cu}$ concentration. Antagonism between $\mathrm{Cu}$ and $\mathrm{Zn}$ might play an important role in pathological processes; it stimulates a shift in metabolism of fat acids and synthesis of prostaglandins [24].

Wide variations are also observed at the level of membrane processes. The content of $\mathrm{K}$ decreases, as cardiac insufficiency progresses, and this tendency undoubtedly influences membrane permeability. As this takes place, $\mathrm{Ca}$ is accumulated in the tissue, thus affecting myocardium contractility (Figure 6). Calcium and potassium metabolic imbalance followed by elongation of the action potential and the associated increase in dispersion of the repolarization process brings about dis- turbances of conductivity and rhythm.

Energy intensity for $\mathrm{Ca}$ inactivation considerably exceeds energy demand for its delivery to myofilaments. A decrease of $\mathrm{Cr}$, as $\mathrm{CHF}$ develops (see Figure 2), is indicative of energy imbalance in the myocardium, therefore, when an increase of intercellular concentration of $\mathrm{Ca}$ is not balanced by increased activity of $\mathrm{Na} / \mathrm{Ca}$ pump, overload of $\mathrm{Ca}$ in the diastole might lead to a postdepolarization phenomenon, trigger activity and eventually to the development of life-threatening ventricular arrhythmias [25].

Of special interest are interrelations between the elements considered as chemical analogs that can replace each other in biochemical reactions (Table 1).

Depending on the functional capabilities of LV myocardium the relationships of these element concentrations change by several times. As cardiac insufficiency aggravates, there occurs a statistically significant reduction of $\mathrm{K}$ content, with $\mathrm{Rb}$ content remaining practically the same, which allows for determining this process as accumulation of $\mathrm{Rb}$ relative to $\mathrm{K}$. It is necessary to note that being a heavier chemical element $\mathrm{Rb}$ is a less desirable one for metabolism.

The relationship of $\mathrm{Ca} / \mathrm{Sr}$ in the case of a decrease in LV contractility results in a statistically significant increase, almost by 2 times, primarily because of $\mathrm{Ca}$ accumulation, with $\mathrm{Sr}$ concentration staying invariable, which can be described as $\mathrm{Sr}$ deficit relative to $\mathrm{Ca}$.

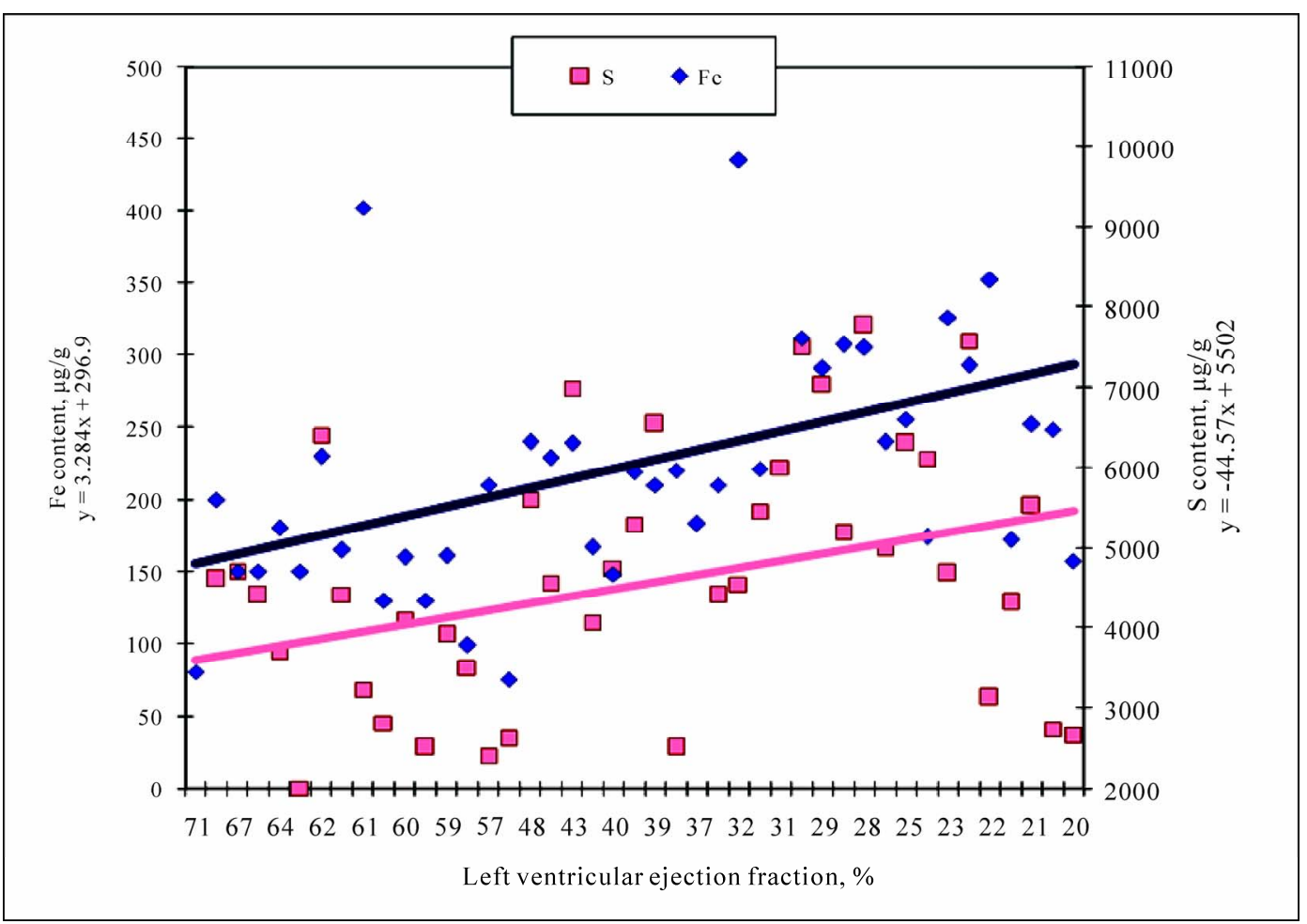

Figure 4. Dynamics of Fe and S content as cardiac insufficiency progresses in CHD patients. 


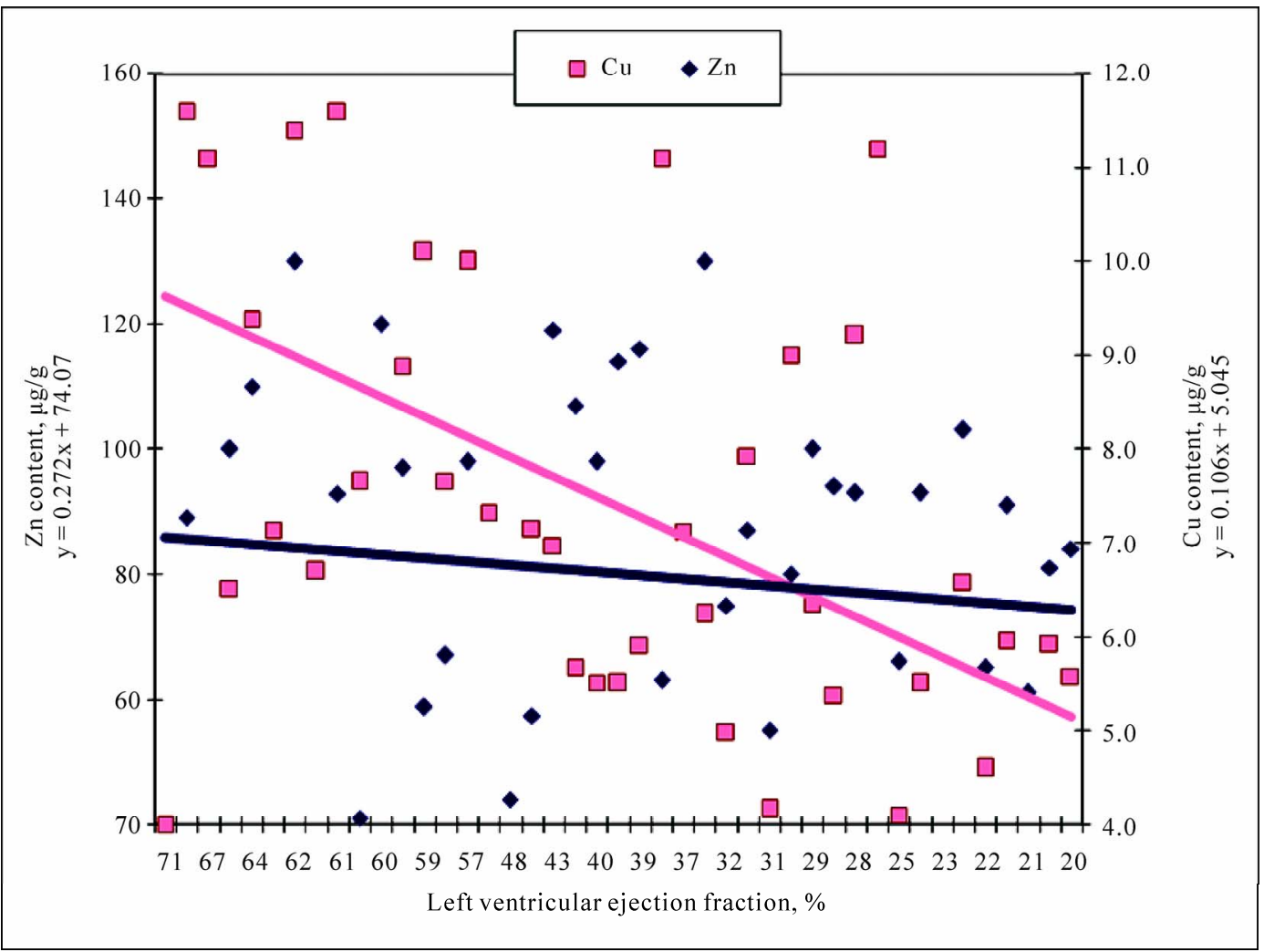

Figure 5. Dynamics of $\mathrm{Zn}$ and $\mathrm{Cu}$ content as cardiac insufficiency progresses in $\mathrm{CHD}$ patients.

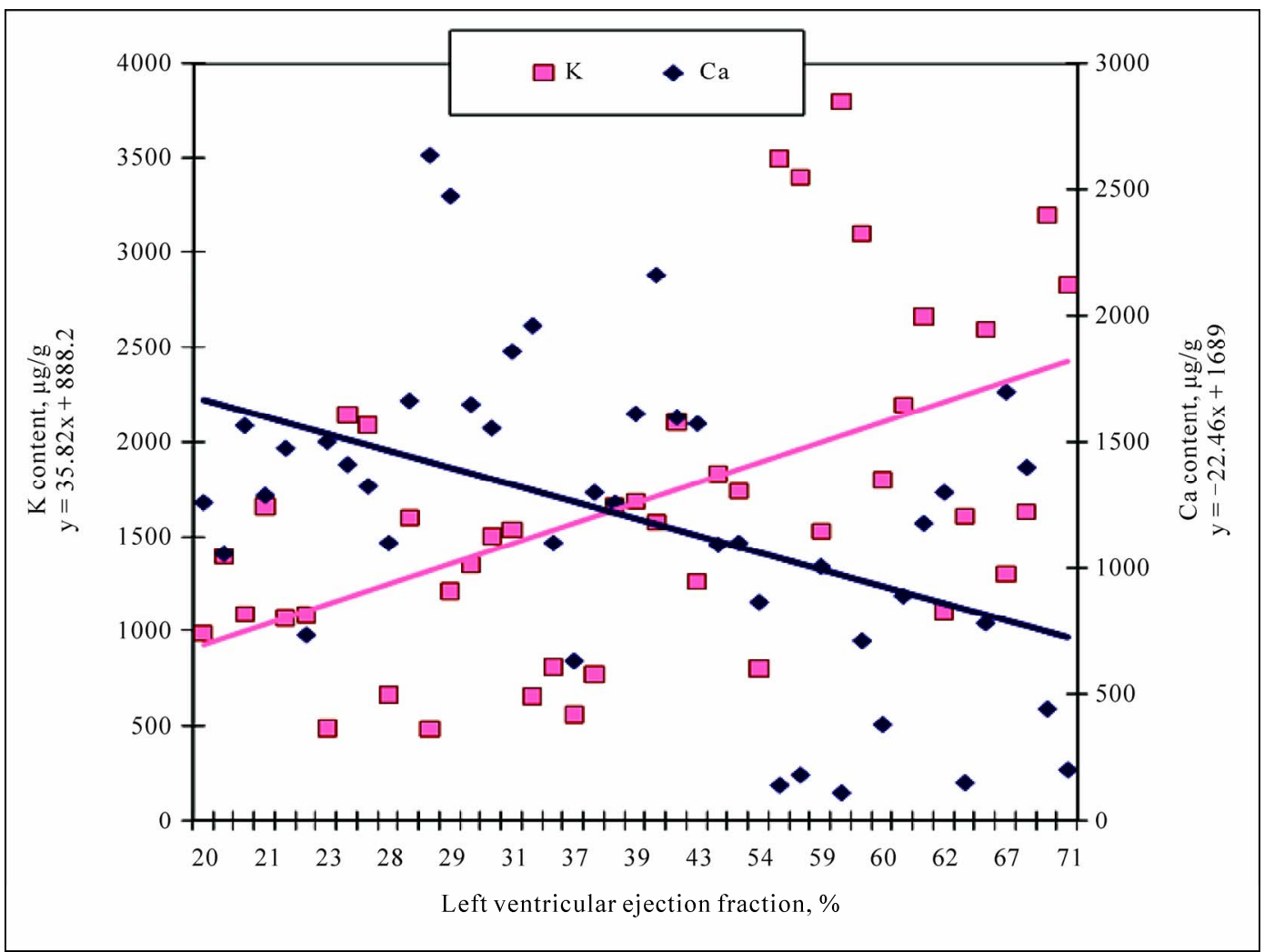

Figure 6. Dynamics of $\mathrm{Ca}$ and $\mathrm{K}$ content as cardiac insufficiency progresses in $\mathrm{CHD}$ patients. 
Table 1. Relationships of some chemical elements in the myocardium of CHD patients with normal and low contractility of $\mathrm{LV}(\mathrm{M} \pm \mathrm{SD})$.

\begin{tabular}{|c|c|c|}
\hline & $\mathrm{K} / \mathrm{Rb}$ & $\mathrm{Ca} / \mathrm{Sr}$ \\
\hline $\begin{array}{l}\text { Patients with Normal LV } \\
\text { Ejection Fraction }(63.5 \pm 1.79 \%)\end{array}$ & $1859 \pm 458.2$ & $369 \pm 69.6$ \\
\hline $\begin{array}{l}\text { Patients with Low LV Ejection } \\
\text { Fraction }(31.2 \pm 1.53 \%)\end{array}$ & $919 \pm 87.8^{*}$ & $701 \pm 69.6^{*}$ \\
\hline
\end{tabular}

The role of $\mathrm{Se}$ in human metabolism is evaluated largely by its concentration in blood, which, as a rule, decreases in the case of a disease. This tendency makes many researchers regard Se deficit as an alarm signal in the development of one or another pathogenesis [26-28]. Even in their first studies some authors attempted to assess the impact of Se on dystrophic processes in muscle tissue [29]. They have found out that Se concentration in blood decreases, as myotic dystrophy develops. However, we have failed to find the works which would explain what is happening to Se concentration in humans with a different functional state of muscle tissue.

Unfortunately, in our study we were also unable to evaluate the contribution of Se to the development of functional dysfunction of LV myocardium in the process of CHF progression due to the absence of a clear relationship of this element content with EF $(r=0.23$; $p>$ $0.05)$. It can be only noted that no statistically significant differences in Se content in the myocardium of CHD patients and the control group have been revealed $(0.78 \pm$ 0.067 и $0.96 \pm 0.056 \mu \mathrm{g} / \mathrm{g}$ of tissue respectively). In literature it is suggested that selenium is very much the same as sulphur. At the same time in living organisms these two elements behave in a completely different way. Specifically, in animals Se compounds tend to regenerate, while $\mathrm{S}$ compounds are subject to oxidation. Selenium is capable of repairing disulfide links in proteins in $\mathrm{SH}$ groups.

We have also found out that there exists a statistically significant correlation relationship of Se content with $\mathrm{Mn}(\mathrm{r}=0.34 ; \mathrm{p}<0.05)$ and with $\mathrm{Zn}(\mathrm{r}=0.44 ; \mathrm{p}<0.01)$, as well as with $\mathrm{Rb}(\mathrm{r}=-0.45: \mathrm{p}<0.01)$ and $\mathrm{Sr}(\mathrm{r}=0.40$; $\mathrm{p}<0.05$ ), which may be explained by free radical oxidation.

Thus, the structural/functional changes accompanying CHF progression are associated with wide variations of metabolic processes in the myocardium, which could be evaluated by the content of chemical elements in tissue. With clinical data on the severity of heart failure, we can assume the status of metabolic processes in the myocardium. Study results on the contents of chemical elements in the myocardium during CHF progression is a fundamental basis for drug development and new approaches in the therapy of heart failure. The results obtained demonstrate that in order to understand the pathogenesis of cardiac insufficiency progression, we need further research in this direction.

\section{REFERENCES}

[1] Focused Update (2009) ACCF/AHA guidelines for the diagnosis and management of heart failure in adults: A report of the American College of Cardiology Foundation/American Heart Association Task Force on practice guidelines: Developed in collaboration with the International Society for Heart and Lung Transplantation. Circulation, 119, 1977-2016.

[2] Ratmanova, A. (2009) Cardiovascular morbidity and lethality statistics in European countries (2008). Medicine Review, 1, 6-12.

[3] Sato, Y., Kita, T., Takatsu, Y. and Kimura, T. (2004) Biochemical markers of myocyte injury in heart failure. Heart, 90, 1110-1113. doi:10.1136/hrt.2003.023895

[4] St. John Sutton, M.G. and Sharpe, N. (2000) Left ventricular remodeling after myocardial infarction. Circulation, 101, 2981-2988.

[5] Kassiri, Z., Zhong, J., Guo, D., et al. (2009) Loss of angiotensin-converting enzyme 2 accelerates maladaptive left ventricular remodeling in response to myocardial infarction. Circulation: Heart Failure, 2, 446-455. doi:10.1161/CIRCHEARTFAILURE.108.840124

[6] Rashed, M.N., Ahmed, M.M., Al-Hossainy, A.F. and Abd, S.M. (2010) Trends in speciation analysis of some heavy metals in serum of patients with chronic hepatitis $\mathrm{C}$ and chronic hepatitis B using differential pulse adsorptive stripping voltammetric measurement and atomic absorption spectrophotometry. Journal of Trace Elements in Medicine and Biology, 24, 138-145.

doi:10.1016/j.jtemb.2009.11.006

[7] Siddiqui, M.K.J., Jyoti, S., Mehrotra, P.K., Singh, K. and Sarangi, R. (2006) Comparison of some trace elements concentration in blood, tumor free breast and tumor tissues of women with benign and malignant breast lesions: An Indian study. Environment International, 32, 630-637. doi:10.1016/j.envint.2006.02.002

[8] Miner, E.C. and Miller, W.L. (2006) A looc between the cardiomyocytes: The extracellular matrix in heart failure. Mayo Clinic Proceedings, 81, 71-76. doi:10.4065/81.1.71

[9] ASSC/SECI (2010) National recommendations on diagnostics and treatment of CHF (3rd revision). Heart Failure, 55, 3-62.

[10] Zile, M.R. and Brutsaert, D.L. (2002) New concepts in diastolic dysfunction and diastolic heart failure: Part I diagnosis, prognosis, and measurements of diastolic function. Circulation, 105, 1387-1393. doi:10.1161/hc1102.105289

[11] Ramani, G.V., Uber, P.A. and Mehra, M.R. (2010) Chronic heart failure: Contemporary diagnosis and management. Mayo Clinic Proceedings, 85, 180-195. doi:10.4065/mcp.2009.0494

[12] Beltrami, C.A., Finato, N., Rocco, M., Feruglio, G.A., et al. (1994) Structural basis of endstage failure in ischemic cardiomyoparthy in humans. Circulation, 89, 151-163.

[13] Novelli, E.L.B., Diniz, Y.S., Machado, T., Proen, B., et al. (2000) Toxic mechanism of nickel exposure on cardiac 
tissue. Toxic Substance Mechanisms, 19, 177-187. doi:10.1080/107691800300119374

[14] Okuneva, G.N., Levicheva, E.N., Loginova, I.Yu., et al. (2009) Myocardium dynamics in health and transposition of the great arteries. Pediatry G.N. Speransky Journal, 87, 29-33.

[15] Patterson, K.Y. and Veillon, C. (2001) Stable isotopes of minerals as metabolic tracers in human nutrition research. Experimental Biology and Medicine, 226, 271-282.

[16] American Diabetes Association (2004) Nutrition principles and recommendations in diabetes (position statement). Diabetes Care, 27, S36-S46. doi:10.2337/diacare.27.2007.S36

[17] Cefalu, W.T. and Hu, F.B. (2004) Role of chromium in human health and in diabetes. Diabetes Care, 27, 2741-2751. doi:10.2337/diacare.27.11.2741

[18] Chowdhury, S., Pandit, K. and Roychowdury, P. (2003) Role of chromium in human metabolism, with special reference to type 2 diabetes. Journal of the Association of Physicians of India, 51, 701-705.

[19] Diastolic Heart Failure (2004) Challenges of diagnosis and treatment. American Family Physician, 69, 2609-2617.

[20] Arredondo, M. and Nunez, M.T. (2005) Iron and copper metabolism. Molecular Aspects of Medicine, 26, 313327. doi:10.1016/j.mam.2005.07.010

[21] Bie de, P., Sluis van de, B., Klomp, L. and Wijmenga, C. (2005) The many faces of the copper metabolism protein MURR1/COMMD1. Journal of Heredity (Special Issue), 96, 803-811.

[22] Palmer, B.M., Vogt, S., Chen, Z., Lachapelle, R.R. and Lewinter, M.M. (2006) Intracellular distributions of es- sential elements in cardiomyocytes. Journal of Structural Biology, 155, 12-21. doi:10.1016/j.jsb.2005.11.017

[23] Tubek, S. (2007) Selected zinc metabolism parameters and left ventricle mass in echocardiographic examination in primary arterial hypertension. Biological Trace Element Research, 118, 138-145. doi:10.1007/s12011-007-0021-0

[24] Campbell, J.D. (2004) Alzheimer's disease: Minerals and essential fatty acids. Journal of Orthomolecular Medicine, 19, 173-182.

[25] Zaugg, C.E. and Buser, P.T. (2001) When calcium turns arrhythmogenic: Intracellular calcium handling during the development of hypertrophy and heart failure. Croatian Medical Journal, 42, 24-32.

[26] Zachara, B.A., Gromadzińska, J., Wasowicz, W. and Zbróg, Z. (2006) Red blood cell and plasma glutathione peroxidase activities and selenium concentration in patients with chronic kidney disease: A review. Acta Biochimica Polonica, 53, 663-677.

[27] Lu, J., Berndt, C. and Holmgren, A. (2009) Metabolism of selenium compounds catalyzed by the mammalian selenoprotein thioredoxin reductase. Biochimica et Biophysica Acta (BBA), 1790, 1513-1519.

[28] Lescure, A., Deniziak, M., Rederstorff, M. and Krol, A. (2008) Molecular basis for the role of selenium in muscle development and function. Chemistry \& Biodiversity, 5, 408-413. doi:10.1002/cbdv.200890041

[29] Orndahl, G., Rindby, A. and Selin, E. (1982) Myotonic dystrophy and selenium. Acta Medica Scandinavica, 211, 493-499. doi:10.1111/j.0954-6820.1982.tb01988.x 